\title{
La diplomacia ciudadana de las fundaciones y organizaciones internacionales en el ámbito migratorio en México
}

\section{The citizen diplomacy of foundations and international organizations in the field of migration in Mexico}

doi: https://doi.org/

10.32870/eees.v26i75.6583

\author{
José Ascención Moreno Mena* \\ Reynaldo Angulo Cázares"
}

\section{Resumen}

El presente artículo analiza la diplomacia ciudadana de las fundaciones y organizaciones civiles internacionales en el territorio mexicano, particularmente en el ámbito del tema migratorio. Mediante seis estudios de caso, con entrevistas a líderes de algunas organizaciones civiles internacionales, se describe cómo las actividades que desarrollan estas organizaciones internacionales tienen la intención, directa o indirecta, de incidir en las políticas públicas internas de México y en las buenas prácticas del Gobierno estadounidense en materia de política migratoria. Se muestra que estas organizaciones desarrollan procesos transnacionales y transfronterizos de gestión pública en alianza con actores no gubernamentales para influir en los Gobiernos. Se sostiene que esas actividades se convierten en diplomacia ciudadana.

Palabras clave: organizaciones civiles, fundaciones internacionales, diplomacia ciudadana, políticas migratorias, México.

\begin{abstract}
This article analyzes citizen diplomacy conducted by international foundations and civil organizations in Mexican territory, dealing with migration issues in particular. Through six case studies, in which some of international civil organizations representatives are interviewed, it's described how activities conducted by these international organizations search for influence, directly or indirectly, internal public policies in Mexico, as well as United States government best practices regarding migration policy. To achieve that, they develop public management transnational and transborder processes in alliance with nongovernmental actors.We claim that these actions become citizen diplomacy.
\end{abstract}

Keywords: Civil organizations, international foundations, citizen diplomacy, migration policies, Mexico.

\footnotetext{
- Profesor-Investigador del Instituto de Investigaciones Sociales de la Universidad Autónoma de Baja California (UABC) campus Mexicali, México. ORCID: http://orcid.org/0000-0002-59621456 jmoreno@uabc.edu.mx

- Profesor-Investigador del Instituto de Investigaciones Sociales de la Universidad Autónoma de Baja California (UABC) campus Mexicali, México. ORCID: http://orcid.org/0000-0002-90631425 reynaldo.angulo.cazares@uabc.edu.mx

Fecha de recepción: 06 de marzo de 2017. Fecha de aceptación: 21 de febrero de 2019.
} 


\section{Introducción}

En México, entre los actores de la sociedad civil que intervienen en el tema migratorio, se pueden encontrar algunos transnacionales como las fundaciones y organizaciones civiles internacionales que llevan a cabo acciones de financiamiento e investigación. Por lo regular, estos actores internacionales se vinculan con organizaciones civiles mexicanas y tienen un efecto visible en sus políticas, prácticas y desempeño. En esa relación, las fundaciones y organizaciones civiles internacionales impulsan procesos transnacionales y transfronterizos de gestión pública, especialmente respecto a los derechos humanos de los migrantes, para incidir en los actores gubernamentales, lo que establece una diplomacia ciudadana.

Este trabajo tiene el propósito de analizar algunos casos de diplomacia ciudadana de fundaciones y organizaciones internacionales en el territorio mexicano. Se sostiene que las acciones que llevan a cabo estos actores internacionales tienen la intención, directa o indirecta, de incidir en las políticas públicas internas del país y en las buenas prácticas del Gobierno estadounidense en materia de política migratoria. Si bien no es pretensión del presente trabajo juzgar estas acciones como positivas o negativas, no se debe dejar de lado que las organizaciones civiles internacionales no solamente se mueven por razones humanitarias o de índole puramente solidaria, sino que sus acciones también tienen una fuerte carga cultural y política que transmite los valores de sus países de origen.

El artículo se centra en la actividad de tres organizaciones civiles estadunidenses que han desplegado una serie de acciones tendientes a incidir en las políticas recientes sobre el tema migratorio: The Washington Office in Latin American (wola), American Friends Services Committee (AFSC) y American Civil Liberties Union (ACLU). Para ello, 
se parte de entrevistas a sus líderes, complementadas con información bibliográfica. También se describe la acción de tres fundaciones internacionales en territorio mexicano: Fundación Appleseed, Fundación Oxfam y Alianza para las Migraciones en Centroamérica y México (CAMminA).

En una primera parte, se aborda el concepto de diplomacia ciudadana. Luego, se conceptualiza a las organizaciones civiles y a las fundaciones internacionales. Posteriormente, se establecen las relaciones entre las organizaciones civiles internacionales y las organizaciones mexicanas. Finalmente, se presentan los tres ejemplos de las fundaciones internacionales que actúan en el ámbito de los temas migratorios en México, así como los tres de las organizaciones civiles estadounidenses elegidas que desarrollan acciones de incidencia en este país.

\section{La diplomacia ciudadana en el contexto de la globalización}

Los diversos procesos de la globalización que comenzaron a experimentarse en el mundo, dice Serbin (2010), contribuyeron al debilitamiento de los Estados nacionales y a un incremento de los flujos transnacionales de diversa índole. Entre los procesos globalizantes, se encuentra el surgimiento de una sociedad civil global y heterogénea que impulsó una nueva visión de la agenda internacional centrada en la defensa de bienes públicos comunes como el desarrollo social, la equidad, el medio ambiente, los derechos humanos y la paz (Serbin, 2010). En este panorama, según Picas (2001), las organizaciones civiles internacionales empezaron a tener un renovado protagonismo internacional al ser convocadas por el Banco Mundial en 1982 para estudiar el papel que deberían desempeñar en el contexto del nuevo modelo económico que se estaba aplicando a escala global, y que afectaría a la mayor parte de los países en desarrollo. 
En el ámbito regional, señala Thorup (1995), la transnacionalización ${ }^{1}$ de la participación cívica fue una de las novedades durante la década de los noventa, y por ello surgió una amplia literatura sobre los procesos de alianza entre organizaciones estadounidenses y latinoamericanas que comenzaron a ser estudiadas por diversos autores, como Chalmers (1992) y Keck y Sikkink (1992) (citados ambos por Thorup -1995-), Heredia y Hernández (1996) y Ghils (1992). Estos trabajos tenían por objeto ver más allá del trabajo de las élites gubernamentales y privadas, es decir, ver cómo actores no gubernamentales de Estados Unidos se interesaban por asuntos como el medioambiente y los derechos humanos en México.

Señala Thorup:

[...] con el paso del tiempo, esas modalidades de participación ciudadana y el creciente fortalecimiento de las organizaciones civiles y sus lazos en ambos países tenían el potencial de modificar las tradicionales asimetrías de poder en la relación de gobierno entre México y Estados Unidos. (Thorup, 1995, p. 163)

En la academia, se empezó a detectar una nueva forma de incidir en las agendas de los países desde la ciudadanía organizada, en espacios donde antes sólo tenían acceso los actores gubernamentales. A esa nueva forma se le comenzó a definir como diplomacia ciudadana. El concepto fue acuñado desde los años cincuenta en diferentes sectores de Estados Unidos "para referirse a la acción de los individuos de contribuir a la conformación de la política exterior de un país[,] diferente a la diplomacia oficial o gubernamental" (Serbin, 2010, p. 116).

I.Por transnacionalización ciudadana se entiende en este trabajo la construcción de redes internacionales de organizaciones civiles, que incluye individuos y grupos de interés comunes, que desarrollan acciones de derechos humanos, medioambiente, comercio, etc., a través de las fronteras de los países. 
En los años ochenta, Joe Montville acuñó el término track two diplomacy o diplomacia de segunda vía para referirse a aquella situación en que ciudadanos particulares u organizaciones fuera del sistema gubernamental intervenían en temas que normalmente estaban reservados para las negociaciones oficiales (Davidson y Montville, 1981).

Davidson y Monteville (1981) mencionan que la diplomacia de segunda vía funcionaba, por ejemplo, cuando había conflictos empantanados, esto es, en situaciones en las que las comunicaciones oficiales y diplomáticas entre los países o entre un Gobierno y un grupo beligerante se habían roto. En este caso, los contactos no oficiales podrían allanar el camino y desacelerar un conflicto antes que cualquiera negociación oficial pudiera hacerlo. Los contactos no oficiales podrían construir puentes entre los actores, aumentar la confianza y fomentar la comprensión mutua.

Algunos lustros después, Diamond y McDonald (1996) desarrollaron el término multi-track diplomacy o diplomacia multivía para abarcar la complejidad y diversidad que progresivamente adquiría la diplomacia de segunda vía, y también para explicar que, además de los canales oficiales, existen otras maneras de desactivar conflictos. Los autores modelaron su idea en un diagrama donde presentaron nueve vías (tracks) (Figura 1), las cuales conforman lo que ellos denominaron círculo de la prevención de conflictos o de la cultura de paz (Diamond y McDonald, 1996). 
Figura 1. La diplomacia ciudadana: el sistema multivías (multi-track)

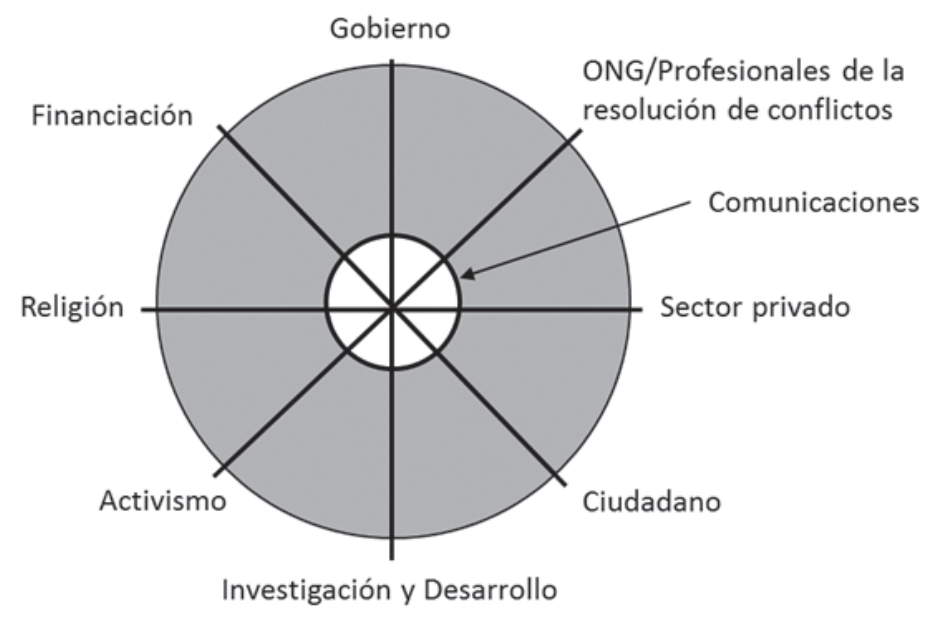

Fuente: Diamond y McDonald (1996, citado en Paffenholz y Spurk, 2006, p. 22).

La primera vía del modelo de Diamond y McDonald está constituida por la diplomacia gubernamental u oficial, tanto nacional como internacional. A los Gobiernos les corresponde en dicha vía un papel de liderazgo e impulso, con las capacidades civiles y militares que poseen. Esta vía es la única que tiene el uso legítimo de la fuerza armada (Diamond y McDonald, 1996). En la segunda vía, aparecen las non profit organizations profesionales (organizaciones sin fines de lucro), dedicadas a los procesos de resolución de conflictos. En la tercera, se encuentra el sector privado, que puede contener actores que contribuyan como agentes de paz.

En la cuarta vía, se encuentran el ciudadano de a pie, que, como tal, puede influir cada vez más -mediante el proceso democrático o su activismo- en la forma en que los Gobiernos y las organizaciones internacionales desarrollan 
su diplomacia. En la quinta vía, se encuentran los esfuerzos internacionales de investigación, educación y capacitación para el desarrollo; aquí se encuentran representados los sectores académico y educativo, por su aporte a las otras vías y a las sucesivas generaciones de ciudadanos en términos de ciencias y cultura de la paz y de la educación.

La sexta vía está constituida por el activismo del ciudadano organizado en redes capaces de influir en los Gobiernos y con potencial de proponer y generar políticas públicas en beneficio de la colectividad. La séptima vía está constituida por los contactos e intercambios entre líderes y seguidores religiosos, comunidades espirituales o confesionales que manifiestan actitudes hacia el pacifismo y la no violencia. En la octava vía se encuentran los esfuerzos de financiación internacional, donde se toma en cuenta que todo proceso necesita tener un sustento, lo que a su vez hace viable que las demás vías puedan operar.

Finalmente, en la novena vía, Diamond y McDonald (1996) incluyen en un punto intermedio entre las organizaciones no gubernamentales (ONG) y el sector privado a la opinión pública y la importancia de la comunicación, de las tecnologías de la comunicación y, muy particularmente, de los medios de comunicación, que los autores consideran que se han convertido en un agente con derecho propio en la gestión internacional de conflictos, pues conectan al resto de vías.

Para Thorup (1995), la diplomacia ciudadana revela las acciones que desarrollan los ciudadanos agrupados en organizaciones civiles de un país para involucrarse en los asuntos de otro. Implica la usurpación de papeles considerados del dominio exclusivo de los actores gubernamentales, es decir, de la diplomacia tradicional.

Otro autor concibe la diplomacia ciudadana como

[...] el esfuerzo sistemático por promover los intereses de diversos sectores de la sociedad civil regional, a través de negociaciones y el 
monitoreo de los acuerdos, en foros multilaterales y procesos internacionales donde hasta ahora tenían acceso exclusivo los diplomáticos oficiales de los Estados que conforman la comunidad internacional de naciones. (Morales, 20I0, p. I)

Serbin, por su parte, propone un concepto más acorde con la realidad latinoamericana:

[Diplomacia ciudadana es] el derecho y la capacidad que requieren materializar y desarrollar las organizaciones ciudadanas, para penetrar en espacios que tradicionalmente han sido privativos de organismos internacionales, gubernamentales y de cooperación externa[,] en función [tanto] de estrategias de cabildeo, negociación e incidencia como de denuncia, movilización y cuestionamiento de estos actores que predo$\mathrm{mina}[\mathrm{n}]$ en el contexto latinoamericano de las redes $y$ organizaciones de la sociedad civil y de los movimientos sociales que aspiran a incrementar su influencia sobre Gobiernos, organizaciones multilaterales y agencias de cooperación internacional. En esta última acepción[,] la diplomacia ciudadana responde a las actuaciones que llevan a cabo actores no gubernamentales por su propia cuenta, sin articularse o coordinarse con la diplomacia oficial desarrollada por los Estados a través de sus embajadores o funcionarios especializados, configurando una diplomacia paralela. (Serbin, 2010, p. II8)

Por su parte, el Foro de Diplomacia Ciudadana, reunido en México en el 2002, definió este concepto en una perspectiva regional como:

[...] el conjunto de esfuerzos, tanto de cabildeo como de negociación [...], de denuncia y de movilización, dirigidos a incidir en ciertas instancias de Gobierno y en los organismos multilaterales por parte de las organizaciones de la sociedad civil, en función de un esfuerzo por democratizar las relaciones internacionales y los foros multilaterales $y$ contribuir a la prevención y resolución de conflictos armados o violentos que puedan emerger en la región. (Serbin, 2010, p. 122) 
2. Las organizaciones civiles internacionales, las fundaciones y sus estrategias de incidencia

Como la diplomacia ciudadana implica la presencia de actores no gubernamentales, este trabajo hace referencia a las organizaciones civiles internacionales (OCI) y a las fundaciones internacionales (FI) que actúan en México. Se entiende como fundación, en términos jurídicos, a una organización constituida sin fines de lucro, que destina parte de su patrimonio al alcance de fines de interés general. Este tipo de instituciones puede estar constituido por personas tanto físicas como morales, sean estas públicas o privadas. Por lo regular, se gobiernan por patronatos o consejos, que tienen el objetivo de cumplir los fines de interés general. Las fundaciones se nutren de las aportaciones, legados y donaciones de sus socios, o bien reciben rentas que genera su propio patrimonio.

Fernández (1995) sostiene que, por lo regular, las fundaciones tienen dos grandes funciones: 1) canalizar recursos económicos en forma de donativos a individuos, organizaciones no lucrativas u otras entidades similares, y 2) proporcionar servicios, realizar investigaciones, organizar conferencias y llevar a cabo publicaciones.

Charry y López (2004), a su vez, distinguen entre fundaciones privadas y comunitarias. Las primeras son creadas por donantes privados o particulares, los cuales pueden ser empresarios o donantes independientes, y tienen un consejo que determina en qué se van a distribuir los recursos. Las fundaciones comunitarias, en cambio, son organizaciones 
públicas formadas por un grupo de individuos para ayudar o beneficiar a su comunidad o región. Por lo regular, los dos tipos de fundaciones son canalizadores de recursos a instituciones no lucrativas, individuos o Gobierno, y buscan elevar la calidad de vida de una zona.

En el caso de las fundaciones que se estudian en el presente artículo, estas provienen de países distintos al que pretenden canalizar recursos, especialmente de países desarrollados. Aquí conviene tener presente el concepto de agentes de difusión (Heydemann y Hammack, 2009), el cual plantea que las fundaciones no sólo llevan a cabo las actividades señaladas previamente, sino que difunden, en los países receptores, lógicas institucionales, entendidas como arreglos organizacionales diseñados "para poner ideas en acción y para sostener patrones de relaciones sociales" (Heydemann y Hammack, 2009, p. 7). Las lógicas institucionales "traen consigo, explícita o implícitamente, tanto intencionalmente como de otra forma, atributos y cualidades que traen la marca de sus lugares de origen" (Heydemann y Hammack, 2009, p. 7).

En este sentido, hay que señalar que muchas de las fundaciones de los países desarrollados fueron creadas con la intención de extender la visión occidental de democracia, desarrollo y filosofía política. Es el caso de las fundaciones alemanas ligadas a partidos políticos de su país, como la Fundación Friedrich Ebert, vinculada al Partido Socialdemócrata, o la Fundación Konrad Adenauer, relacionada con el Partido Demócrata Cristiano. Otras fundaciones tienen su origen en las Iglesias, ya sea cristiana o católica, que pretenden llevar la caridad a las comunidades más pobres, desarrollar acciones humanitarias y promover la cultura de la paz, pero también extender sus creencias. Algunas fundaciones de ese tipo son Manos Unidas, Intermon-Oxfam, Misereo, Trocaire, Cáritas y Sociedad Religiosa de Amigos Cuáqueros, entre otras. También están las fundaciones nor- 
teamericanas, que nacieron para promover los valores de la democracia estadounidense, la modernización tecnológica, un medioambiente sustentable, los derechos humanos y el estilo de vida americano (american way of life).

En lo que respecta a organizaciones operativas, en este trabajo se hace referencia a las oci. Canto (2004) las define como una "organización libre, voluntaria, de ciudadanos, que a partir de la identificación sobre campos específicos de la vida social realiza acciones tendientes al bienestar colectivo, para lo cual pretende influir en las decisiones públicas y en su normatividad" (p. 57). Esta definición rescata los objetivos que se proponen las ocI, como los vinculados a sus fines humanitarios y sociales y la aplicación del concepto de incidencia pública. También permite observar que las ocI son entidades privadas independientes de las instituciones gubernamentales y los organismos públicos. El financiamiento de estos grupos proviene de diversas fuentes, entre ellas, subvenciones y concursos de organismos públicos, donaciones de fundaciones y entidades privadas, aportación de los integrantes, venta de servicios y actividades de recaudación de fondos. Incluso, muchas organizaciones civiles reciben recursos provenientes de los Gobiernos de sus países de origen.

De esta manera, las fundaciones y las organizaciones civiles están estrechamente relacionadas, pues ambas pertenecen al ámbito de la sociedad civil y buscan beneficiar a terceros, aunque por lo regular las oci son financiadas por las fundaciones para que desarrollen sus proyectos. Vistas así, las OCI son operativas y las fundaciones canalizan recursos económicos. Las oci, al igual que las Fi, no están exentas de la influencia de los intereses de sus respectivos países.

En cuanto a la participación de las ocI, Ghils (1992) menciona tres formas en que estas suelen intervenir en los asuntos de los Estados-nación. La primera es en la adopción de nuevas normas internacionales, donde la sociedad civil 
organizada internacional se convierte en grupo de presión. La segunda puede ser la acción directa de las oci en el plano transnacional, a favor ya sea de sus miembros o de grupos sociales particulares, especialmente los que están en una situación vulnerable. Finalmente, la tercera se refiere a las intervenciones que se presentan en ámbitos en que los Gobiernos no intervienen o son incapaces, y en los que las organizaciones civiles desafían abiertamente el orden estatal o interestatal.

Las formas de intervención que tienen mayor impacto son aquellas en las cuales las organizaciones civiles nacionales se enlazan con otras similares de distintos países y conforman redes o coaliciones transnacionales para intentar influir en las agendas de las llamadas cumbres $y$ conferencias de discusión internacional, realizadas por la Organización de las Naciones Unidas (ONU), en las cuales presentan informes sobre la situación prevaleciente en sus países (Avendaño, Moreno, y Priego, 2000). Al respecto, dice Cruickshank que "para que la diplomacia ciudadana sea efectiva[,] debe tener bases de organización en redes locales, nacionales e internacionales" (2013, p. 22).

Para Calduch Cervera (1991), las acciones de influencia o presión ejercidas por las OCI hacia los Estados no siempre deben entenderse exclusivamente en un sentido negativo, pues con frecuencia los Gobiernos locales se benefician de y apoyan la colaboración que les brindan muchas de estas agrupaciones, pues sus recursos humanos y materiales y su actuación internacional permiten paliar o complementar las deficiencias y limitaciones que poseen los distintos países.

En síntesis, la diferencia entre las fundaciones y las OCI estriba en que las primeras tienen como fin financiar proyectos y las segundas los llevan a cabo. 


\section{Relaciones entre las organizaciones civiles mexicanas y las estadounidenses}

La presencia de FI y OCI en México data de principios del siglo xx. Sin embargo, no fue sino hasta los años cincuenta cuando surgieron organizaciones comunitarias financiadas por fundaciones ligadas a la Iglesia católica. Estos grupos comenzaban a implementar programas de desarrollo comunitario, especialmente en el campo. Reygadas (1998) menciona que entre ellos se encontraban grupos herederos del anarquismo y grupos de profesionistas universitarios que tenían un compromiso social.

El objetivo de la mayoría de estas organizaciones comunitarias era llevar conocimientos, capacitación y educación mediante el extensionismo ${ }^{1}$ principalmente hacia el sector campesino. Las Fi tenían la visión de que el pueblo mexicano sufría de un atraso importante en relación con los países europeos, y que había que modernizarlo. Con el advenimiento de la llamada revolución verde, aparecieron fundaciones -como la Rokefeller-que entraron en México con su capital para apoyar la modernización del campo mexicano. Lo que en realidad buscaban dichas fundaciones era perpetuar el colonialismo tecnológico (Hewitt de Alcántara, 1978). Posteriormente, en los setenta y ochenta, se establecieron organizaciones de carácter filantrópico o asistencial, que desarrollaban sus actividades en los ámbitos de la salud, la educación y la alimentación de los pobres, aunque estas se encontraban más ligadas a las fundaciones religiosas o entidades que fungían como tales (Cáritas Ciudad de México, 2016).

I. Se define extensionismo como "el servicio prestado por personal de las instituciones de educación y de investigación que facilita el acceso al conocimiento, la información y las tecnologías a productores, grupos y organizaciones económicas rurales y a otros actores del sector agropecuario, pesquero y acuícola" (Ugalde Vitelly, 20I2). 
Durante ese periodo (primeros tres cuartos del siglo xx), las FI financiaron múltiples proyectos de organizaciones civiles mexicanas, desde las que pretendían el desarrollo económico y los derechos humanos, hasta las que pugnaban por la democratización del país. Algunos de los financiadores internacionales presentes fueron las fundaciones Ford, Rockefeller, Nacional para la Democracia, John D. and Catherine T. MacArthur y W. K. Kellogg, así como el Instituto Synergos (Charry y López, 2004; Jiménez, 1990).

A partir del terremoto de 1985 en el centro del país, los fondos internacionales fluyeron de manera importante para el apoyo a múltiples proyectos (Hernández, 1992). En el periodo 1988-1992, las relaciones transnacionales entre actores no gubernamentales de Canadá, Estados Unidos y México se acentuaron durante las negociaciones del Tratado de Libre Comercio con América del Norte (TLCAN).

En el ámbito de la migración, París, Zenteno, Treviño y Wolf (2015) mencionan que en dicho periodo se realizaron esfuerzos de coordinación entre las organizaciones nacionales y las internacionales para la documentación, la investigación aplicada y la incidencia en política nacional e internacional:

Probablemente, el primer esfuerzo en la materia fue la fundación de la Coordinadora Nacional de Organizaciones No Gubernamentales de Ayuda a Refugiados (Conongar), en 1989. Formada por quince organizaciones que trabajaban principalmente en la Ciudad de México con refugiados guatemaltecos y salvadoreños dispersos, la Conongar ocupó de 1992 a 1994 la coordinación de la Mesa de Refugiados Latinoamericanos del Consejo Internacional de Agencias Voluntarias (International Council of Voluntary Agencies -ICVA-), con sede en Ginebra. (París, Zenteno, Treviño, y Wolf, 20I 5, p. 204)

Por su parte, en la década de los noventa, la Fundación Oxfam-Canadá comenzó a trabajar con organizaciones 
civiles mexicanas en el tema del refugio a los migrantes centroamericanos (Thorup, 1995).

En el ámbito trasfronterizo de Estados Unidos y México, se presentaron acercamientos informales entre organizaciones civiles de ambos lados de la frontera que abordaban la temática de derechos humanos y la asistencia a los migrantes. Esto se dio particularmente en la frontera entre San Diego y Tijuana durante la discusión de la entonces iniciativa de Ley Simpson-Mazzoli, entre 1982 y 1983, y posteriormente con la promulgación de la Ley Simpson-Rodino, de 1986 (Avendaño, Moreno, y Priego, 2000). A partir de esos acercamientos se conformaron algunas redes y coaliciones, como la Coalición Hispana para la Defensa de los Derechos Humanos del Condado de San Diego (Thorup, 1995).

Las experiencias y relaciones de redes transfronterizas se han consolidado paulatinamente durante los últimos años. Sobresalen las siguientes redes: la formada por la Coalición Prodefensa del Migrante de Baja California y la American Friends Services Committee (AFSC); la constituida por la American Civil Liberties Union (ACLU) y la Coalición Prodefensa del Migrante de Baja California; la alianza entre ACLU y la Iniciativa Kino en Nogales, Sonora, y Nogales y Douglas, Arizona; y la integrada por la ACLU y la Iniciativa Frontera Norte de México, apoyada por la Fundación Ford, en particular su programa de Defensa e Incidencia Binacional (Del Ángel et al., 2013).

El otro ejemplo de proyectos importantes fue el Foro Migraciones, mencionado por París, Zenteno, Treviño y Wolf (2015), que durante casi quince años estuvo conformado por más de cuarenta organizaciones y académicos y cuyo financiamiento provenía de varias fundaciones extranjeras, entre ellas la Ford. Este foro constituyó un espacio de análisis y discusión de experiencias para generar propuestas que modificaran los marcos normativos que impactaban el fenómeno migratorio, y sobre todo para motivar aquellos que fueran favorables a la 
protección de los derechos humanos de las personas migrantes (París, Zenteno, Treviño, y Wolf, 2015).

Durante ese tiempo, también se presentaron las Conferencias Regionales sobre Migración, en las que participaban los países del TLCAN. Estas tenían la finalidad de discutir políticas en materia de migración, y a ellas acudían las organizaciones civiles, financiadas por FI. Como resultado de estas experiencias, se logró conformar una red de carácter regional que incluía organismos de México, Estados Unidos y Centroamérica, la cual fue nombrada Red Informal sobre Migración.

\section{La participación de las fundaciones internacionales en el tema migratorio de México}

En este apartado, se describen brevemente tres FI que han tenido un protagonismo en el tema migratorio en México y que, desde el punto de vista de los autores del presente artículo, tienen inscritas sus acciones en el concepto de diplomacia ciudadana.

La primera es la Fundación Appleseed, establecida en Estados Unidos en 1993 por un grupo de abogados de la Universidad de Harvard que pretendían ofrecer asesoría jurídica gratuita a iniciativas sociales que buscaran cambios estructurales. La Fundación Appleseed tiene diecisiete centros en Estados Unidos y México, y se presenta como libre de ideologías o afiliación política y con la única finalidad de lograr el bien común. En su página de México, se menciona que desarrolla asesoría jurídica a organizaciones civiles para su fortalecimiento, identifica causas sociales que requieran análisis legal con el propósito de introducir recomendaciones y planes de acción para generar cambios sistémicos que beneficien a los sectores más vulnerables, y promueve e institucionaliza la práctica del trabajo probono en México (Appleseed, 2016). 
Fundación Appleseed se involucró en el tema migratorio primero en Estados Unidos, para lo cual desplegó una campaña de información para las comunidades de inmigrantes indocumentados asentadas en territorio estadounidense y sujetas a deportación, de modo que estas supieran cómo proteger a sus familias y cómo recuperar sus bienes desde México. Para ello, tuvo que desarrollar un diálogo con las autoridades de ambos países.

En México, Appleseed-México, con el apoyo de Unicef, desarrolló una investigación y mapeo en el 2003 sobre las condiciones del derecho a la identidad en el territorio mexicano, con la finalidad de presentar recomendaciones e iniciativas legislativas y de política pública al Gobierno mexicano.

En el 2010, Appleseed-Estados Unidos, en colaboración con la Fundación Annie E. Casey, elaboró el Manual de protección de bienes y custodia de menores al enfrentar la deportación. Posteriormente, en el 2011, la misma fundación llevó a cabo en Estados Unidos y México un estudio legislativo y de campo sobre el sistema de expulsión y repatriación de los menores migrantes mexicanos no acompañados. El propósito era mejorar la protección inmediata de niños, niñas y adolescentes detenidos y repatriados a México. Las investigaciones y publicaciones de informes de Appleseed han influido en las buenas prácticas de los Gobiernos de México y Estados Unidos.

Las acciones de la Fundación Appleseed se inscriben perfectamente en la diplomacia ciudadana, en la medida en que provienen de una organización civil que actúa para intentar influir en las políticas sociales de un país distinto al suyo.

La segunda organización tratada es la Fundacion Oxfam, una confederación internacional formada por diecisiete organizaciones que realizan labores humanitarias en noventa países. Fue fundada en 1942 y tiene su sede en Inglaterra. 
Oxfam-México nació como Fundación Vamos en 1996, integrada por líderes y directivos de organizaciones civiles con una larga trayectoria en el campo del desarrollo social y los derechos humanos. En 2005, Vamos se convirtió en Rostros y voces, y posteriormente, en el 2008, se integró a Oxfam Internacional.

Entre los objetivos que se plantea la fundación están construir un futuro libre de la injusticia y la pobreza, mejorar las condiciones y los medios de vida de las personas más vulnerables, fortalecer las organizaciones locales e influir en los Gobiernos y empresas para garantizar derechos. En cuanto al tema migratorio, Oxfam pretende

Fortalecer el protagonismo de las organizaciones de migrantes como actores binacionales, para incidir en las políticas públicas nacionales y regionales, con el objetivo de lograr el pleno reconocimiento de sus derechos y un desarrollo más incluyente. (Oxfam International, 2019)

Hasta ahora, según la propia página de Oxfam, solamente se han desarrollado acciones, campañas, y apoyos económicos para el fortalecimiento de organizaciones civiles de migrantes, sin que se mencionen los logros obtenidos de manera específica, que hacen alusión al apoyo para el establecimiento de un albergue destinado a migrantes centroamericanos en México.

También de manera específica, se hace referencia a un apoyo otorgado gracias a Oxfam en conjunto con la Secretaría de Desarrollo Social a la Coalición Prodefensa del Migrante para la realización de un programa de documentación en derechos humanos de los migrantes deportados en Baja California.

Es posible decir que esta fundación apenas comienza a desarrollar su objetivo, pero es claro que pretende incidir en las políticas públicas nacionales y regionales desarrollando procesos de diplomacia ciudadana. 
La tercera fundación es CAMMINA, fundada en el 2010 por tres instituciones: Fundación Ford, Fundación Avina y las Fundaciones para una Sociedad Abierta. Estas fundaciones en alianza se han propuesto:

- Objetivos: promover y proteger los derechos humanos de las poblaciones migrantes, refugiadas y solicitantes de asilo por medio de la incidencia en políticas públicas relacionadas con los sistemas de políticas migratorias, de acceso a la justicia y al sistema laboral.

- Misión: lograr la adopción y eficaz instrumentación de políticas públicas que promuevan y protejan los derechos humanos, con énfasis en los civiles y laborales; el empleo digno de las poblaciones migrantes, refugiadas y solicitantes de asilo, y sus familias; así como el derecho a no emigrar; generando oportunidades que les permitan tener una vida digna y sostenible en sus comunidades de origen. (CAMMINA, 2017)

Las fundaciones que integran CAMMINA han reunido experiencias, fortalezas y estrategias para encontrar soluciones transnacionales; fortalecer y crear nuevos vínculos entre actores de la sociedad civil, Gobiernos y otras instituciones; y acompañar procesos innovadores y replicables, todo esto en temas de migración.

CAMMINA apoya económicamente a un sinnúmero de organizaciones civiles de la región que trabajan en favor de las personas migrantes, lo que incluye organizaciones civiles mexicanas. Los temas que tienen que ver con la niñez migrante, mujeres migrantes, deportados y refugiados son a los que destina mayor apoyo.

En el 2014, CAMMINA acordó un plan de trabajo con organizaciones aliadas por la niñez y adolescencia migrante de la región, con el fin de contribuir a la consolidación de una estrategia para incentivar el empleo de más de un millón de jóvenes en riesgo de migración y deportados y de otras 
poblaciones en ocho países de la región (Avina, 2016). El financiamiento de CAMMINA está enfocado en la incidencia pública: sus recursos no están destinados a apoyar iniciativas asistenciales, sino a organizaciones y proyectos que busquen transformaciones en los países donde actúan. Por ello, su injerencia también representa una clara acción de diplomacia ciudadana.

\section{La intervención de las organizaciones} civiles internacionales

En los últimos años, organizaciones civiles estadounidenses también se han preocupado por la situación de los migrantes, especialmente de los mexicanos y centroamericanos. En este apartado, se hace referencia a tres ocI con esta característica, cuyos líderes fueron entrevistados.

La primera ocI es wolA. En realidad, es un centro de estudios y promoción de los derechos humanos que tiene su sede en Estados Unidos. Desde su fundación, en 1974, wolA ha jugado un papel importante en los debates políticos que se realizan en los espacios legislativos del país norteamericano sobre los derechos humanos en América Latina. Se considera un interlocutor clave con el Poder Ejecutivo de ese país, en organizaciones multilaterales y representantes del Congreso estadounidense, y es fuente frecuente para la prensa del continente americano. Según se menciona en su página, la misión que se ha propuesto es "lograr un continente en el cual las políticas públicas protejan los derechos humanos y reconozcan la dignidad humana, y en donde la justicia prevalezca sobre la violencia” (WOLA, 2016).

A través de colaboraciones estratégicas, wola trabaja con defensores de derechos humanos, académicos, líderes religiosos, artistas, negocios y actores gubernamentales que pueden incidir en un cambio social y propugnar por sociedades más justas en todo el continente americano. 
WoLA tiene un programa específico para México en el cual se aborda el tema migratorio de manera especial; se documentan y exponen las causas de la violencia en la frontera y en otras zonas del país; y se monitorea la asistencia de seguridad de Estados Unidos hacia México, para evaluar que el financiamiento vaya hacia reformas efectivas al sistema judicial. También, wola provee apoyo a organizaciones mexicanas que buscan la justicia para víctimas de abusos de derechos humanos.

Meyer (2016), investigadora y activista, dirige el programa sobre México en WolA, con un enfoque especial en las políticas de seguridad de dicho país y Estados Unidos y su relación con la violencia asociada al crimen organizado, la corrupción y las violaciones a los derechos humanos en el país centroamericano. Ella coordina el trabajo de woLA sobre la seguridad fronteriza y la migración, y hace trabajo de incidencia para generar mayores protecciones para los migrantes en tránsito en México y en la frontera entre este y Estados Unidos:

El programa sobre México empezó a trabajar el tema de los delitos y violaciones a los derechos humanos de los migrantes en tránsito en 2010 por el aumento de los secuestros. En 20I I, creamos el programa sobre seguridad fronteriza y la migración. Este programa analiza las políticas de seguridad fronteriza de Estados Unidos, su cooperación con México en [la materia] y el impacto de las políticas estadounidenses en el bienestar y los derechos de la población migrante en la frontera México-Estados Unidos, en particular prácticas y políticas que ponen a los migrantes en riesgo. En 2013, expandimos este trabajo para analizar las políticas de seguridad fronteriza y de migración del Gobierno mexicano en su frontera sur, el apoyo financiero y político del Gobierno estadounidense a estas políticas y el impacto del aumento de la infraestructura de seguridad fronteriza y de los esfuerzos para detener y deportar a migrantes en la población en tránsito por México. (Meyer, 2016) 
WOLA se relaciona tanto con sectores gubernamentales tomadores de decisiones en Estados Unidos y México como con organizaciones civiles que trabajan con migrantes:

En Estados Unidos, tenemos una relación con el Departamento de Seguridad Interna (DHS, por sus siglas en inglés), el Departamento de Estado, asesores en la Casa Blanca y asesores en el Congreso. En México, tenemos una relación con el Instituto Nacional de Migración (INM), la Procuraduría General de la República (PGR), la Secretaría de Relaciones Exteriores (SRE) y la Secretaría de Gobernación (Segob). Aunque los representantes de los Gobiernos muchas veces no están de acuerdo con nosotros y nuestros hallazgos, no es una relación de confrontación. Trabajamos en alianza con organizaciones mexicanas y locales en Estados Unidos. (Meyer, 2016)

El valor que le da wola a sus contrapartes en México es fundamental. La diplomacia ciudadana no sería efectiva sin este componente:

WOLA fue establecida para poder dar voz en Washington a colegas en la región, para que los tomadores de decisión puedan escuchar de primera mano las preocupaciones que hay en la región en materia de derechos humanos y el impacto de las políticas y el apoyo de Estados Unidos. WOLA no tendría razón de ser si no fuera por nuestro trabajo con contrapartes en la región. (Meyer, 2016)

La diplomacia ciudadana implica que la oci usurpa temas de la agenda nacional -en este caso, el migratorio- de un Gobierno de un país diferente al suyo para incidir de manera directa o indirecta en sus políticas internas, pero siempre con tendencia a favorecer a los grupos marginados o que no tienen voz:

[...] nuestro rol es presionar a los Gobiernos para que ellos hagan cambios en sus políticas para mejorar el respeto a los derechos humanos en 
sus países, presentar propuestas de políticas para atender a debilidades institucionales que llevan a violaciones a los derechos humanos y a la impunidad,y presentar propuestas sobre cómo mejorar la cooperación de Estados Unidos en la región. Aunque los Gobiernos deben estar vigilando los derechos de los ciudadanos, los ciudadanos tienen que exigir estos derechos y reclamar cuando [los Gobiernos] no los están protegiendo. (Meyer, 2016)

Sin embargo, la diplomacia ciudadana no se circunscribe a influir en los Gobiernos extranjeros, sino también busca incidir en el de origen, en un afán por democratizar las relaciones internacionales y contribuir a la prevención y resolución de conflictos:

Por otro lado, hemos trabajado para apoyar agendas de actores gubernamentales. Un ejemplo de esto es la colaboración que hemos tenido con el Gobierno mexicano para denunciar prácticas estadounidenses de deportación que ponen a los migrantes en riesgo, así como prácticas de CBP [Customs and Border Protection] que buscaron investigar y sancionar a niños mexicanos involucrados en el tráfico de personas o drogas sin contemplar los riegos para los niños o su bienestar. (Meyer, 2016)

En torno a la comprensión de sus acciones, a wola le queda muy claro que su actuación se inscribe en la diplomacia ciudadana:

WOLA sí hace trabajo de diplomacia ciudadana. Por un lado, buscamos incidir en las políticas de nuestro propio país hacia la región, sobre todo en la cooperación en materia de seguridad, y muchas veces estos esfuerzos los hacemos en colaboración con contrapartes en la región. Por otro lado, hacemos trabajo de incidencia directamente con los Gobiernos en la región sobre políticas que ponen en riesgo los derechos de sus ciudadanos o de migrantes, y proponemos cambios en políticas para mejorar diferentes situaciones o para atender fallas 
estructurales en las instituciones de procuración de justicia, seguridad pública y migración. (Meyer, 2016)

Sobre su papel en la diplomacia ciudadana como organización extranjera en México, Meyer (2016) menciona distintas estrategias por las que intenta incidir en los temas migratorios, en alianza ya sea con organizaciones civiles o con académicos:

En México, realizamos trabajo de incidencia para apoyar los esfuerzos de varias organizaciones mexicanas, en particular la Fundación para la Justicia y el Estado de Derecho Democrático, para establecer la Unidad de Investigación de Delitos para Personas Migrantes y el Mecanismo de Apoyo Exterior Mexicano de Búsqueda e Investigación, dentro de la PGR [y] aprobado en diciembre de 20I5. WOLA realiza investigaciones sobre diferentes temas con base en visitas al campo donde realizamos entrevistas con una diversidad de actores, así como la revisión de los medios y documentos oficiales e informes de académicos y [de] otras organizaciones. En temas migratorios, hemos realizado informes conjuntos con instituciones académicas, casas del migrante y organizaciones de derechos humanos. Un ejemplo de esto fue la realización del informe Un camino incierto, publicado en 2015. Aparte de los informes, escribimos documentos cortos sobre temas particulares que distribuimos a nuestros contactos y con los medios, y emitimos boletines de prensa respondiendo a diferentes políticas o eventos. También hemos realizado videos sobre prácticas de deportación que ponen a los migrantes en riego en la frontera [entre] México y Estados Unidos, fallas en la protección de los menores migrantes mexicanos en la frontera, y la falta de protección de la niñez migrante centroamericana en sus países de origen y en México. También realizamos reuniones con tomadores de decisión de Estados Unidos y de México y con asesores en el Congreso de Estados Unidos. (Meyer, 2016)

La segunda ocI que se presenta como caso es la AFSC. Es una organización afiliada a la Sociedad Religiosa de Amigos Cuá- 
queros que promueve ayuda humanitaria. Trabaja por la justicia social, la paz duradera con justicia, la reconciliación, los derechos humanos y la abolición de la pena de muerte. AFSC fue fundada en 1917 por miembros estadounidenses de la Religious Society of Friends y de la asistencia a víctimas civiles de la Primera Guerra Mundial. En 1947, AFSC recibió el Premio Nobel de la Paz.

Entre sus objetivos, la asociación menciona el acompañamiento y capacitación de las organizaciones mexicanas y estadounidenses y la creación de redes que puedan influir directamente en el desarrollo de la comunidad a través de la negociación, la mediación y la transformación de conflictos, en especial respecto a la seguridad fronteriza y su relación con la seguridad nacional.

Pedro Ríos, coordinador de la oficina de AFSc-San Diego, menciona que la organización apoya el crecimiento y desarrollo de las organizaciones dirigidas por migrantes a través del fortalecimiento de una red de comités de derechos humanos que tiene el objetivo de llevar las voces e inquietudes de las comunidades de inmigrantes a los debates sobre políticas a nivel local, estatal y nacional.

El enfoque de nuestra oficina en San Diego ha [buscado] incidir a los tres niveles de Gobierno de los Estados Unidos en el tema migratorio, por medio del trabajo legislativo. A nivel local [región San Diego] se ha logrado ampliar el apoyo a la comunidad migrante a través de resolutivos y proclamaciones a favor de la reforma migratoria justa y humanitaria. A nivel federal, el impacto político es el de incidir en procesos tanto legislativos como administrativos. El enfoque [busca] asegurar que los cuerpos migratorios fronterizos, así como la CBP, tengan mecanismos de mayor transparencia y un sistema de rendimiento de cuentas. (Ríos, 2016)

La AFsC tiene una política más conciliatoria que de confrontación con las autoridades, pero en ocasiones las circunstancias la llevan a situaciones del segundo tipo: 
Incidimos con oficiales públicos y representantes administrativos. Cuando es necesario aumentar la presión pública sobre algún tema de importancia, sí se ha llegado a la confrontación, especialmente en el tema del abuso [protagonizado] por autoridades migratorias y fronterizas. (Ríos, 2016)

Aunque la asociación inscribe sus acciones en la región fronteriza San Diego-Tijuana, sus repercusiones trascienden las fronteras: la AFSC en los últimos años también ha realizado alianzas con organizaciones del lado mexicano, especialmente con la Coalición Prodefensa del Migrante de Baja California, con la cual ha organizado el conversatorio Análisis sobre la conflictividad en la frontera: violencia, seguridad y derechos humanos, en 2014 y 2015. En estos eventos, se buscaba identificar las fuentes generadoras de conflictos en la materia y cómo éstas afectaban a los grupos y personas migrantes. Asimismo, se pretendió que las organizaciones civiles, académicos y funcionarios participantes realizaran un autodiagnóstico sobre sus capacidades y carencias para afrontar dichos conflictos, y que los diferentes sectores representados llegaran a consensos en la identificación de los retos y en la presentación de propuestas, durante y después del evento, para contribuir a la transformación de los conflictos identificados.

Sobre los tipos de recursos que despliega la AFsc para incidir en las políticas públicas de su país, Ríos (2016) menciona:

Las acciones para incidir en el Gobierno de los Estados Unidos incluyen boletines de prensa con declaraciones en respuesta a políticas o situaciones [relacionadas] con el tema de migración; cabildeo [en] los tres niveles de Gobierno, local, estatal, federal; encuentros entre comunidades afectadas; foros públicos e informativos en el Congreso de los Estados Unidos; audiencias con representantes del Congreso; cartas de apoyo [a] o en contra de propuestas de ley; manifestaciones y acciones 
públicas en relación al tema de la migración; diálogo con autoridades de Gobierno; e investigaciones sobre el tema de la migración.

En cuanto a la política de alianzas de la AFSC para incidir tanto en las autoridades estadounidenses como en las mexicanas, el líder señala:

\begin{abstract}
Nuestra organización considera muy importante destacar el trabajo de incidencia con alianzas y agrupaciones que se guían con los mismos objetivos para alcanzar un cambio social. Para esto, conformamos parte de coaliciones al nivel local, estatal y regional (frontera Estados Unidos-México). En algunas ocasiones, la relación de trabajo con ciertas organizaciones nos ha llevado a incidir en foros internacionales sobre el tema migratorio. Estas alianzas son importantes para poder tener el mayor impacto posible con los recursos unidos de varias organizaciones de la sociedad civil. Demuestra[n] a las autoridades que el respaldo [o rechazo] de una propuesta de ley o medida administrativa es apoyado por una alianza amplia de la sociedad civil. (Ríos, 2016)
\end{abstract}

Recientemente, la AFSC estableció alianzas en territorio mexicano con la Coalición Prodefensa del Migrante para elaborar el informe Vidas en la incertidumbre. La migración forzada hacia la frontera norte de México zy nuestra solidaridad? En el 2016, el mismo se presentó en el ámbito nacional (México), lo que permitió desplegar toda una campaña para que el Gobierno mexicano reconociera el problema del desplazamiento interno y actuara creando un marco normativo en materia de protección de desplazados. Sus acciones tuvieron resultados parciales con la emisión de medidas cautelares que hizo la Comisión Nacional de Derechos Humanos (CNDH) al INM, autoridades del Gobierno de Baja California y al presidente municipal de Tijuana, con la intención de que coordinaran acciones conjuntas para la atención de las personas migrantes solicitantes de asilo que llegan a Baja California (Notimex, 2016). 
Sobre la percepción en torno a la diplomacia ciudadana, el líder de la AFsc comenta:

[...] nuestras acciones representan el resultado de un proceso de diálogo con las comunidades migrantes del condado de San Diego, y entonces la incidencia por nuestra parte es una extensión de la diplomacia ciudadana, por lo menos representando al nivel local desde una región fronteriza. (Ríos, 2016)

Aunque Ríos no habla de la incidencia transfronteriza que su organización ha intentado desplegar, dado que se refiere a la AFSC de San Diego, existen múltiples ejemplos en los que su organización ha incursionado en territorio mexicano y desde ahí influido en la política estadounidense. Tal es el caso de las protestas por los asesinatos de algunos migrantes como Anastasio Hernández Rojas, que murió en 2010 luego del exceso de violencia utilizado por agentes fronterizos en la garita de San Ysidro (Truax, 2010), o la presentación del mencionado informe sobre migrantes desplazados en el centro de México. En el último ejemplo, el texto menciona:

AFSC promueve esfuerzos que incluyen trabajar con las autoridades y con la sociedad civil para mejorar las políticas públicas nacionales que permitan la generación de condiciones nacionales y regionales para garantizar de manera sostenible el cumplimiento de los derechos primordiales de las personas migrantes; acceso a servicios integrales, medidas/soluciones que aborden las causas de raíz, etc. (Avendaño, Moreno, y Siu, 2016, p. II)

La tercera OCI que se toma como caso es la ACLU. Esta es una organización sin fines de lucro surgida en Estados Unidos, en 1920. Tiene oficinas en cincuenta estados de la Unión Americana, aunque las centrales se encuentran en Nueva York. Su misión es defender y preservar los derechos indi- 
viduales y las libertades garantizadas a cada persona por la constitución y las leyes de Estados Unidos.

Esmeralda Flores, coordinadora de algunos programas fronterizos de la ACLU en San Diego, describe cómo está estructurada la organización:

Nuestro trabajo se realiza en los tribunales y las legislaturas para cambiar leyes que nos impactan a todos. A pesar de que la ACLU es una organización nacional, cada afiliado tiene áreas de trabajo diferentes, según la comunidad en la que trabaja. La ACLU de San Diego constantemente colabora con organizaciones en México. Muchas veces trabajamos en colaboración con otras oficinas en el estado o en campañas nacionales. Las protecciones constitucionales fundamentales del debido proceso y la igualdad de protección consagradas en nuestra constitución y declaración de derechos se aplican a todas las personas, independientemente de su condición migratoria. La protección de estos derechos en contra de cualquier forma de discriminación es parte fundamental de nuestro trabajo a favor de la comunidad migrante, desde lo local hasta el nivel nacional. (Flores, 2016)

Al igual que todas las organizaciones civiles, la ACLU despliega un repertorio de recursos para cumplir sus fines, en este caso en el ámbito fronterizo de San Diego-Tijuana:

En ACLU San Diego, los derechos de las personas migrantes son de gran importancia. Nuestro equipo realiza pláticas Conoce tus derechos [para preparar a los migrantes cuando deban] interactuar con la policía, con [la] patrulla fronteriza y con ICE [Us Immigration and Customs Enforcement, o Servicio de Inmigración y Control de Aduanas], con el objetivo de que la población migrante sepa cuáles son sus derechos y cuándo un agente está actuando dentro de su competencia. Además, tenemos el Programa de Litigio Fronterizo, que tiene como objetivo documentar las violaciones en que la patrulla fronteriza y CBP incurren. ACLU tiene [también] el Proyecto de Derechos de Inmigrantes a nivel nacional basado en San Francisco. Dependiendo de la situación en particular, 
ACLU puede realizar investigaciones y reportes, conferencias de prensa, artículos de opinión para periódicos, cabildeo a favor o en contra de ciertas propuestas de leyes, o demandas para cambiar ciertas políticas, prácticas o leyes. (Flores, 2016)

En su política de alianzas, se contemplan organizaciones gubernamentales locales y extranjeras, defensores de derechos humanos de la sociedad civil y otros actores:

Para el tema de migración, constantemente estamos interactuando con el Departamento de Seguridad Interna [DHS, por sus siglas en inglés], con ICE, patrulla fronteriza y CBP. Muchas de estas interacciones se realizan de manera contenciosa, pero de manera general se trata de crear y mantener una relación cordial con las autoridades, sobre todo a nivel local. Realizamos alianzas con otros defensores de derechos de migrantes, con organizaciones de trabajadores y con organizaciones de justicia racial. ACLU trabaja específicamente en los Estados Unidos, pero al trabajar el tema de la migración es necesario colaborar con organizaciones o entidades de Gobiernos extranjeros. Es importante crear alianzas porque agregan fuerza al movimiento social,ya que muchas veces existe una intersección en estas tres áreas de justica social y es importante demostrar cómo la migración no es tema aislado de otros. (Flores, 2016)

En torno a la percepción sobre la diplomacia ciudadana, Flores reconoce que no todas las acciones se pueden llevar a cabo en territorio estadounidense, sobre todo cuando se trata de los derechos humanos de los migrantes, por lo que la asociación se ve obligada por las circunstancias a desarrollar diplomacia ciudadana:

Al trabajar en el tema de la migración, sobre todo desde un estado y ciudad fronterizos, es imposible negar que se necesita trabajar en ambos lados de la frontera para que las políticas y prácticas sean recíprocas, coherentes y efectivas. En la dinámica de la migración, se necesita trabajar en los países receptores, los de tránsito y los expulsores. (Flores, 2016) 
En torno a la diplomacia ciudadana transfronteriza, cabe destacar una de las acciones a través de la cual la ACLU ha logrado incidir más en Estados Unidos a favor de la comunidad migrante desde el territorio mexicano: su movilización respecto al llamado acuerdo López-Venegas, un litigio ganado en las cortes de Estados Unidos en contra de ICE y la patrulla fronteriza. ${ }^{2}$ A través de este acuerdo, personas que fueron injustamente deportadas podrían regresar a Estados Unidos para recibir el debido proceso y tener una oportunidad de pelear por permanecer con sus familias. La implementación del acuerdo López-Venegas requirió por primera vez que ACLU de San Diego trabajara en México más allá de Baja California.

Para el impulso al acuerdo señalado, la ACLU desplegó una serie de estrategias de difusión en medios tradicionales y redes sociales. Se establecieron relaciones con distintos actores: en Baja California con el Consejo Estatal de Atención al Migrante, la delegación de la SRE, la CNDH y la Coalición Prodefensa del Migrante; y en el ámbito nacional, con la misma SRE - para la realización de foros informativos binacionales dirigidos a la sociedad civil e instancias de Gobierno-, la Red Consular en Estados Unidos y la Coordinación Nacional de Oficinas de Atención al Migrante (Conofam). Igualmente, la ACLU se apoyó en otras organizaciones civiles mexicanas como Mexicanos y Americanos Todos Trabajando, Instituto de las Mujeres en la Migración, el Centro de Derechos del Migrante Inc., el Colectivo de las Migraciones para América y Radio Chiapaneco.

En resumen, la asociación realizó un total de veinte foros presenciales, una conferencia de prensa en Ciudad de

2. El acuerdo López-Venegas incluyó disposiciones que permitieron a algunos de los miles de mexicanos que han sido expulsados de los Estados Unidos a través de salidas voluntarias ilícitas reunirse con sus familias en dicho país. 
México y otra en Tijuana, y una presentación ante el Senado de la República mexicano, con bastante éxito. ${ }^{3}$

\section{Conclusiones}

Uno de los objetivos de este trabajo fue demostrar que las FI y las oci desarrollan diplomacia ciudadana en materia migratoria en México. Se propuso, además, enriquecer esta perspectiva ampliándola, volviéndola más realista para el estudio de relaciones asimétricas entre países, como las que históricamente han prevalecido entre México y Estados Unidos.

Se señaló que, aunado al proceso de diplomacia ciudadana, las organizaciones estudiadas despliegan, velada o directamente, visiones e intereses de sus países de origen. Es decir, estas organizaciones pueden ser vistas como agentes de difusión que promueven estrategias alineadas a intereses de sus naciones y, muy importante, que proyectan formas de organización en los países receptores de la asistencia. Frente a un escenario de vulnerabilidad social, esto último no siempre es negativo, más aún cuando se promueven y defienden los derechos humanos y se busca avanzar en la democratización y empoderar a los actores sociales desfavorecidos.

En el caso de las Fi y ocı que operan en México y que incorporaron el tema migratorio en sus agendas, estas nacieron en el extranjero en medio de otros objetivos nacionales, pero se percataron de que el fenómeno migratorio rebasaba sus fronteras y de que sus causas se encontraban también en otros territorios, así que para poder incidir en una transformación y encontrar alternativas que favorecieran los intereses de su país y que previnieran los conflictos tenían que buscar incidir en las políticas públicas de Gobiernos ajenos.

3. Una corte federal aprobó el acuerdo en febrero de 2015.

\section{0}


Para esa incidencia, era necesario que las asociaciones intervinieran en las agendas de discusión, elaboraran diagnósticos, participaran en espacios de negociación, hicieran sus propias propuestas -en ocasiones en contraposición a la documentación estatutaria oficial-, dieran seguimiento a sus propuestas y presionaran mediante sus alianzas nacionales.

En el caso concreto de las FI, sus actividades de apoyo están encaminadas fundamentalmente a programas y organizaciones que pretenden incidir en las políticas e instituciones de los países expulsores de migrantes.

En cuanto a las ocI, estas tienen más libertad para moverse de un territorio a otro y establecer alianzas con organizaciones que tienen intereses comunes. Continuamente asisten u organizan foros concernientes a la migración, y participan en foros paralelos donde se presentan informes oficiales o en visitas de organismos internacionales -como la ONU o la Comisión Interamericana de Derechos Humanos (CIDH)-, donde colaboran en sus informes. Aunque la mayoría de las organizaciones y FI manifiestan ser muy respetuosas de las políticas de los países donde desarrollan sus actividades, y en ese sentido son funcionales a los Gobiernos nacionales en turno, en la práctica no están exentas de atravesar obstáculos y conflictos con los actores gubernamentales.

$\mathrm{Al}$ respecto de lo anterior, el caso de wolA resulta emblemático. Si bien su misión principal explícita es la promoción de los derechos humanos en las Américas (en realidad, tiene en mente a América Latina), se detecta, además de lo anterior, el impulso de otros objetivos no explícitos, como la seguridad fronteriza (border security) con México, la contención de la violencia en toda la región y el freno de la migración hacia los Estados Unidos. En realidad, la promoción de los derechos humanos aparece como subproducto del freno a la migración para la asociación. Esto resulta más marcado cuando se trata del caso de los migrantes de países 
de América Central, mientras que en el caso de México son asuntos prioritarios para WolA la promoción de la seguridad fronteriza, el combate a las drogas y la contención de la violencia, esto último sobre todo en la frontera norte de México. En todo momento, se ve reflejado en los programas de la organización (transparencia, rendición de cuentas, etc.) que el interés estadounidense es prioritario.

A su vez, también el interés fundamental de CAMMINA es contener la migración, especialmente de jóvenes centroamericanos y mexicanos que se encuentran en condiciones precarias. El propósito de la ACLU y de la Fundación Appleseed, por su parte, es propiciar un Estado de derecho a imagen y semejanza del estadounidense, mientras que el de AFSC es prevenir los conflictos que se originan por situaciones de pobreza y violencia en los países menos desarrollados. El propósito de la Fundación Oxfam es construir los medios para abatir la injusticia y la pobreza, así como empoderar a los actores nacionales que puedan incidir en el mejoramiento de las condiciones de vida de su país.

$\mathrm{Al}$ revisar las políticas y programas que estas organizaciones dicen impulsar y, en menor medida, implementar, es de esperarse que sólo se pueda apreciar su proyección y no su adopción voluntaria por parte de las organizaciones y actores mexicanos con quienes trabajan. Desde una perspectiva crítica, sería más enriquecedor ir más allá de la declaración explícita de sus objetivos o metas, pues esta sólo sería una primera capa, quizás la más superficial. Aun así, lo que sí puede apreciarse es la consistencia y coherencia en las políticas y en los arreglos institucionales que estas organizaciones promueven en la región, sintetizadas en las llamadas reformas comprensivas: protección y promoción de los derechos humanos, reformas a los aparatos judiciales y policiacos para que respeten los derechos individuales, rendición de cuentas, Estado de derecho, entre otras. Estudios futuros sobre este tema harían una contribución mayor si 
profundizaran en los contenidos de las políticas y acciones de las asociaciones en la región.

Bibliografía

Appleseed (2016). Nuestro trabajo. Recuperado de http:// www.appleseedmexico.org/

Avendaño, R. M., Moreno,J.A.,y Priego, E. (2000). Las ONG en la frontera Baja California-California: un acercamiento al estudio de la diplomacia ciudadana. Estudios fronterizos, I (I), 89-I 35.

Avendaño, R. M., Moreno, J. A., y Siu, E. (2016). Vidas en la incertidumbre: la migración forzada mexicana hacia la frontera norte de México iy nuestra solidaridad? Tijuana: Coalición Prodefensa del Migrante, American Friends Service Committee. Recuperado de https://www.afsc. org/sites/default/files/documents/Vidas\%20en\%20la\%20 Incertidumbre.pdf

Avina (20I6). Un millón de migrantes podrán ser beneficiados por estrategia intersectorial impulsada por CAMMINA. Avina. Recuperado de http://www.avina.net/avina/un-millon-de-migrantes-podran-ser-beneficiados-por-estrategia-intersectorial-impulsada-por-CAMMINA-2/

Calduch Cervera, R.(1991). Relaciones internacionales. Madrid: Ediciones Ciencias Sociales.

CAMMINA (20I7). Nosotros. Recuperado de https://cammina. hiponline.org/es/nosotros

Canto, M. (2004). La disputa teórica sobre las organizaciones civiles. Un asunto político-ideológico. En J. Cadena (comp.), Las organizaciones civiles mexicanas (pp. 49-72). México: Universidad Nacional Autónoma de México, Centro de Investigaciones Interdisciplinarias en Ciencias y Humanidades.

Cáritas Ciudad de México (2016). Antecedentes institucionales e historia. Recuperado de http://www.caritas-mexico. org.mx/index.php/quienes-somos-w/antecedentes-ins- 
titucionales-e-historia/360-antecedentes-instituciona-

Bibliografía les-e-historia

Charry, C. I., y López, S. (2004). Las fundaciones comunitarias en México y el mundo. Polis: investigación y análisis sociopolítico y psicosocial, 2(4), 9-45.

Cruickshank, S. (20I3). La diplomacia ciudadana en los noventa: espacios multilaterales como espacio de interacción ciudadanía-Gobiernos. Revista mexicana de política exterior, (98), 19-47.

Davidson,W.D.,y Montville,J.V. (198I). Foreign Policy According to Freud. Foreign Policy, (45), I45-I57. doi https:// doi.org/ I 0.2307/I I 483 I 7

Del Ángel, P., et al. (20।3). Violaciones a derechos humanos de personas migrantes mexicanas detenidas en los Estados Unidos 20II-20I2. Segundo informe del Programa de Defensa e Incidencia Binacional de la Iniciativa Frontera Norte de México. México: PDIB. Recuperado de https:// programadefensaincidenciabinacional.files.wordpress. com/2012/05/2do-informe-violaciones-a-ddh-de-mexicanos-deportados-de-usa.pdf

Diamond, L., y McDonald, J. ( 1996). Multi-Track Diplomacy. A Systems Approach to Peace. Connecticut: Kumarian Press. Fernández, R. M. (coord.) (1995). Perfil de las fundaciones en México. México: Cemefi, Fundación Ford.

Flores, E. (22 de octubre de 2016). Entrevista en comunicación personal en su calidad de coordinadora de asociación [J. Moreno, entrevistador]. Tijuana, México.

Ghils, P. (1992). La sociedad civil internacional: las organizaciones internacionales no gubernamentales en el sistema internacional. Revista internacional de ciencias sociales, (I33), 443-458.

Heredia, C., y Hernández, R. (I 996). La diplomacia ciudadana en la era de la globalización: un punto de vista desde México. México: DECA, Equipo Pueblo, A. C. 
Bibliografía
Hernández, L. (I I de noviembre de 1992). La nueva cooperación internacional a través del espejo: notas sobre la diplomacia ciudadana en la década de los noventa [ponencia presentada en la VII Reunión de Profmex-ANUIES]. Mérida, México.

Hewitt de Alcántara, C. (1978). La modernización de la agricultura mexicana: 1940-1970. México: Siglo XXI.

Heydemann, S., y Hammack, D. C. (2009). Philanthropic Projections: Sending Institutional Logics Abroad. En D. C. Hammack, y S. Heydemann (eds.), Globalization, Philanthropy, and Civil Society: Projecting Institutional Logics Abroad (pp. 3-3I). Bloomington: Indiana University Press. Jiménez, M.A. (1990). La Fundación Rockefeller y la investigación agrícola en América Latina. Comercio exterior, 40(10), 968-975.

Meyer, M. (I7 de noviembre de 2016). Entrevista en comunicación personal en su calidad de líder de asociación [J. Moreno, entrevistador]. Ciudad de México, México. Morales, R.A. (2010). ¿Qué es la diplomacia ciudadana? Parte I.Recuperado de http://aich2008.blogspot.mx/20 I0/04/ que-es-la-diplomacia-ciudadana.html.

Notimex (I8 de septiembre de 2016). CNDH pide medidas cautelares en garita de Tijuana ante ola migrante. $\mathrm{La}$ Jornada Baja California. Recuperado de http://jornadabc. $\mathrm{mx} /$ tijuana/l8-09-20 I6/cndh-pide-medidas-cautelares-en-garita-de-tijuana-ante-ola-migrante

Oxfam International (2019). México. Recuperado de https:// www.oxfam.org/es/paises/mexico?fbclid=IwAR IUhcXXJNq-E0MwzMy6zf2QR _IWucfclcaoFTqHHI5Tt5ehStJO60P5I-M

Paffenholz, T., y Spurk , C. (2006). Civil Society, Civic Engagement, and Peacebuilding. Social Development Papers. Conflict Prevention and Reconstruction, (36). Recuperado de http://siteresources.worldbank.org/INTCPR/Resources/WP36_web.pdf 
París, M. D., Zenteno, R., Treviño, J., y Wolf, S. (20I5). Un Bibliografía análisis de los actores políticos y sociales en el diseño y la implementación de la política y la gestión migratoria en México. Tijuana: El Colegio de la Frontera Norte, Fundación Ford.

Picas, J. (200 I). Papel de las organizaciones no gubernamentales y la crisis del desarrollo. Una crítica antropológica a las formas de cooperación [tesis doctoral inédita]. Doctorado en Antropología Social, Universidad de Barcelona: Barcelona. Recuperada de http://hdl.handle.net/10803/705

Reygadas, R. (1998). Abriendo veredas. Iniciativas públicas y sociales de las redes de organizaciones civiles. México: Convergencia de Organismos Civiles por la Democracia. Ríos, P. (I I de octubre de 20 I6). Entrevista en comunicación personal en su calidad de líder de asociación [J.Moreno, entrevistador]. San Diego, California.

Serbin,A. (2010). La diplomacia ciudadana en América Latina y el Caribe: una actualización. Política externa, 18(4), II5-I30.

Thorup, C. L. (1995). Diplomacia ciudadana, redes y coaliciones transfronterizas en América del Norte: nuevos diseños organizativos. Foro internacional, $x x x v(2),|55-2| 8$. Truax, E. (03 de junio de 20 I0). Homicidio en el caso Anastasio Hernández. La Opinión. Recuperado de https:// www.pressreader.com/usa/la-opinión2078/20100603/28 I 487862586903

Ugalde Vitelly, G. (26 de junio de 20I2). El extensionismo rural en México. Inforural. Recuperado de https://www. inforural.com.mx/el-extensionismo-rural-en-mexicol WOLA (2016). Programa Migración y Seguridad Fronteriza. Recuperado de https://www.wola.org/es/taxonomy/term/77I 